\title{
Relevance of the JAK2V617F mutation in patients with deep vein thrombosis of the leg
}

\author{
Mandy N. Lauw • Erik W. N. Bus • Alexander F. Y. van Wulfften Palthe • \\ Michiel Coppens • Christa H. Homburg • Saskia Middeldorp • \\ C. Ellen van der Schoot • Harry R. Koene • Bart J. Biemond
}

Received: 7 January 2011 / Accepted: 28 March 2011 / Published online: 12 April 2011

(C) The Author(s) 2011. This article is published with open access at Springerlink.com

\begin{abstract}
Venous thromboembolism (VTE) can be the first presenting symptom in myeloproliferative neoplasms (MPN). Studies have demonstrated a high prevalence of the JAK2V617F mutation in patients with splanchnic vein thrombosis. Fewer studies have been done in patients with thrombosis outside the splanchnic area, showing a lower prevalence although the clinical relevance of the mutation in these patients, e.g., progression to overt MPN, remains unknown. The objective of this study was to determine the effect size of JAK2V617F in prospectively collected DNA samples of patients objectively diagnosed with deep vein thrombosis (DVT) of the leg and controls without DVT, with follow-up on JAK2V617F-positive patients to assess clinical relevance. Presence of JAK2V617F was determined in DNA samples from 187 patients with DVT and 201 controls, using quantitative RT-PCR. Hematological parameters were also analyzed. All initially JAK2V617F-positive patients were reassessed. Of 187 patients with DVT, 178 were analyzed for JAK2V617F, and in four $(2.3 \%$; $95 \%$ CI 0.1-4.4), JAK2V617F was present. Of 201 controls, 198
\end{abstract}

\footnotetext{
M. N. Lauw $\cdot$ H. R. Koene $\cdot$ B. J. Biemond $(\triangle)$

Department of Hematology, Academic Medical Center, F4-224, PO Box 22660, 1100 DD Amsterdam, The Netherlands e-mail: b.j.biemond@amc.uva.nl

E. W. N. Bus $\cdot$ C. H. Homburg C. E. van der Schoot Experimental Immunohematology, Sanquin Research at CLB, Amsterdam, The Netherlands
A. F. Y. van Wulfften Palthe • M. Coppens · S. Middeldorp Amsterdam, The Netherlands Department of Vascular Medicine, Academic Medical Center,

H. R. Koene

Department of Hematology, St. Antonius Hospital, Nieuwegein, The Netherlands
}

were analyzed; one was JAK2 $2617 \mathrm{~F}$ positive $(0.5 \%$; $95 \%$ CI $-0.5-1.5$, OR 4.5 ; 95\% CI 0.5-40.9). None had MPN features, nor upon reassessment after a median follow-up of 68.5 months. Four JAK2V617F-positive patients with DVT and one control without DVT did not develop overt MPN after a median follow-up of nearly 6 years. Thus, in patients with non-splanchnic venous thrombosis, JAK2V617F appears not to be clinically relevant.

Keywords Venous thrombosis - JAK2V617F mutation . Janus kinase-2 mutation · Myeloproliferative neoplasms . Clinical relevance

\section{Introduction}

Venous thromboembolism (VTE) is a major cause of morbidity and mortality in Philadelphia mutation-negative myeloproliferative neoplasms (MPN). In 12-49\% of cases, VTE can manifest years before the disease becomes clinically apparent [1]. MPN-related VTE characteristically concerns deep vein thrombosis (DVT), pulmonary embolism, occlusion of the cerebral veins, or of the splanchnic veins, e.g., hepatic (Budd-Chiari syndrome), portal, splenic, or mesenteric veins.

In 2005, the JAK2V617F mutation was discovered that can be found in $>1 \%$ of cells in up to $97 \%$ of patients with polycythemia vera $(\mathrm{PV})$, and in $50 \%$ of patients with essential thrombocythemia (ET), and primary myelofibrosis (PMF) [2-4]. Analysis of JAK2V617F has been incorporated in the diagnostic criteria for MPN [5], and quantitative analysis can also be used for monitoring of disease response [6, 7]. JAK2V617F seems to be absent in the healthy population [8], although some studies have found a very low prevalence in healthy subjects [9-11], resulting in 
the hypothesis that the presence of JAK2V617F in healthy cohorts may be a first indication of an underlying MPN.

Several studies have addressed the association between JAK2 $2617 \mathrm{~F}$ and thrombosis in the absence of MPN. JAK2 $2617 \mathrm{~F}$ is strongly associated with splanchnic vein thrombosis (SVT) and has been demonstrated in $40-60 \%$ of patients with Budd-Chiari syndrome [12-14] and 25-50\% of patients with portal vein thrombosis [15-17]. In patients with venous thrombosis outside the splanchnic area, the prevalence of JAK2V617F was low $(0.0-2.0 \%)$ [18-22]. However, the clinical relevance of the mutation in this group, e.g., progression to MPN and therapeutic consequences, remains unclear, as no studies to assess the risk and timing of progression to an overt MPN have been published so far.

Therefore, we addressed the effect and clinical relevance of JAK2 $2617 \mathrm{~F}$ in prospectively collected DNA samples of patients diagnosed with DVT of the leg, and in a cohort of patients in whom DVT was objectively ruled out (controls). Hematological parameters were analyzed retrospectively. Follow-up was performed on all patients who harbored the JAK2V617F mutation upon initial determination, to assess clinical relevance based on progression of the mutated allele burden and hematological parameters.

\section{Materials and methods}

Identification of patients and controls

DNA samples were obtained from consecutive patients of at least 18 years old who were referred for suspicion of acute symptomatic DVT to the Academic Medical Center in Amsterdam, The Netherlands, between September 1999 and May 2006. The criteria for DVT were a proximal leg vein that was not compressible on ultrasonography or presence of an intraluminal filling defect on venography. Proximal DVT was defined as a thrombus in the popliteal vein, superficial femoral vein, or common femoral vein. If compression ultrasonography showed no venous thrombosis and the D-dimer plasma level was $\geq 0.5 \mathrm{mg} / \mathrm{L}$, compression ultrasonography was repeated after 7 days. DVT was ruled out in case of a Wells score [23] $\leq 1$ in combination with a low D-dimer plasma level $(<0.5 \mathrm{mg} / \mathrm{L})$. DVT was also ruled out in case of a normal venography or negative compression ultrasound in combination with a low D-dimer plasma level $(<0.5 \mathrm{mg} / \mathrm{L})$, and after a repeated negative ultrasound.

We selected 187 DNA samples of consecutive patients with objectively diagnosed DVT and 201 DNA samples of patients without previous VTE, with a clinical suspicion of
DVT but in whom DVT was objectively excluded (controls), matched individually for age ( \pm 5 -year intervals) at the time of blood sample collection. Patients with DVT were selected irrespective of previous VTE, presence of thrombophilia, or provoking co-morbidities. Patients with MPN at the time of DVT diagnosis were excluded. Hematological parameters were examined retrospectively using patient records. DNA was isolated from leukocytes in peripheral blood using standard procedures and stored in refrigerated cabinets $\left(4^{\circ} \mathrm{C}\right)$ under anonymous codes. Blood samples were taken after written informed consent, and the presence of clinical risk factors for VTE was assessed using a structured questionnaire. Collection of DNA was approved by the Medical Ethical Committee of the Academic Medical Center, Amsterdam, The Netherlands, for prospective exploration of unknown risk factors of VTE and in accordance with the Declaration of Helsinki.

Our follow-up strategy implied that all patients, who harbored the JAK2V617F mutation upon initial determination, were re-evaluated in the beginning of 2009. Patients were informed about the presence of JAK2V617F in their DNA sample and possible consequences. Also, information about this study was given. After informed consent, blood samples were taken to determine hematological parameters and to assess JAK2V617F.

\section{Real-time quantitative TaqMan PCR}

The presence of the JAK2V617F mutation was assessed using a highly sensitive allele-specific real-time quantitative TaqMan PCR assay, performed on an ABI 7900HT Fast Real-Time PCR System (Applied Biosystems, Foster City, CA, USA). After initial denaturation $\left(20 \mathrm{~s}\right.$ at $\left.95^{\circ} \mathrm{C}\right) \mathrm{PCR}$ was carried out for 50 cycles at fast conditions $\left(1 \mathrm{~s} 95^{\circ} \mathrm{C}\right.$, $20 \mathrm{~s} 60^{\circ} \mathrm{C}$ ). TaqMan Fast Universal Master Mix (Applied Biosystems) was used; primers (300 $\mathrm{nM}$ each) and probe (200 nM) were as designed by Kröger et al. [7] and purchased from Eurogentec (Liege, Belgium). The final concentration is $300 \mathrm{nM}$ for the primers and $200 \mathrm{nM}$ for the probe. The DNA input is $100 \mathrm{ng}$. Samples were run in duplicate. As described by Quentmeier et al. [24], the HELcell line (derived from a patient with AML M6) is positive for the JAK2V617F mutation but has an eightfold tandem duplication of the JAK2-gene. A tenfold dilution of this HEL-cell line DNA is set at $100 \%$. Serial dilution into buffy coat-derived MNC from a pool of ten healthy donors was used as standard curve $(100 \%, 10 \%, 1 \%, 0.1 \%$, and $0.01 \%$ ) for quantification in cases and controls. A RQ-PCR of albumin was performed to calibrate.

The sensitivity of our assay was $0.1 \%$. DNA samples were considered JAK2V617F positive if they demonstrated duplicate positive signals on PCR with cycle threshold $(\mathrm{Ct})<43$. In 
this assay, an allelic burden of $1 \%$ results in a $\mathrm{Ct}$ value of 35 . Samples demonstrating duplicate positive signals with $\mathrm{Ct} \geq 43$ as well as samples demonstrating a single positive signal with $\mathrm{Ct}>40$ on PCR were considered negative for presence of the JAK2V617F mutation. These criteria have been established based on previous testing of a control cohort of healthy volunteers $(n=381)$. Mutated allele burdens were expressed as the percentage of total JAK2 represented by JAK2V617F (percent JAK2V617F) [25].

Outcome measures and statistical analysis

The primary outcomes of the study were the effect of the JAK2V617F mutation on the risk of DVT, calculated as an odds ratio (OR) with corresponding 95\% confidence intervals (CI), and progression to overt MPN in patients who harbored JAK2V617F at initial assessment to assess clinical relevance.

\section{Results}

\section{Patient characteristics}

Of 187 patients with objectively diagnosed DVT, eight DNA samples did not yield sufficient DNA, leaving 179 (95.7\%) for analysis of JAK2V617F and hematological parameters. Of 201 controls without DVT, three samples did not yield sufficient DNA, leaving 198 (98.5\%) for analysis. An evident MPN (PV) was retrospectively diagnosed in one DVT-patient by examination of hematological parameters at the time of DVT diagnosis. This patient was excluded from further analyses. Blood counts in all remaining patients did not fulfill the diagnostic WHO criteria for MPN [5]. Age and gender distribution did not differ significantly between patients with and without DVT.

Of the 178 patients with DVT, $25.8 \%$ had experienced a previous thrombotic episode. In $83(46.6 \%)$, one or more thrombophilic defects were detected (factor $\mathrm{V}$ Leiden heterozygosity $(n=27)$, deficient protein $\mathrm{C}$ or $\mathrm{S}(n=4$ and 2 , respectively), hyperhomocysteinemia $(n=16)$, antiphospholipids syndrome $(n=9)$, elevated factor VIII $(n=30)$, prothrombin 20210A mutation $(n=7)$ ). DVT was provoked by operation, malignancy (treatment), trauma, pregnancy, or use of oral contraceptives in 59 cases (33.1\%). In 43 cases $(24.2 \%)$, no risk factors for thrombosis could be acknowledged.

JAK2V617F mutation; initial determination

In four of $178(2.3 \%$; 95\% CI $0.1-4.4)$ patients with DVT, the JAK2V617F mutation was present using RT-PCR.
Quantitative analysis was possible in one patient (JAK2V617F allele burden $0.87 \%$ ); in the other three cases, the mutational burden was insufficient for quantification. In the control group without DVT, the JAK2V617F mutation was present in one of $198(0.5 \%$; $95 \%$ CI $-0.5-$ 1.5) samples, while the mutational burden was insufficient for quantification. Hematological parameters, age, and gender distribution did not differ significantly between JAK2V617F-positive and negative patients. None of the five JAK2V617F-positive patients had experienced a previous thrombotic episode, and in four of five, DVT occurred without known thrombophilic or provoking factors. Presence of the JAK2V617F mutation increased the risk for DVT 4.5-fold, although not statistically significant (OR 4.5; 95\% CI 0.5-40.9).

\section{JAK2V617F mutation; follow-up}

We reassessed all five patients who harbored JAK2V617F for hematological parameters and JAK2V617F mutational burden. The median follow-up was 68.5 months (range 47 85). Upon reassessment, the JAK $2 \mathrm{~V} 617 \mathrm{~F}$ mutation was present in three of five initially JAK2V617F-positive patients. Quantification of the JAK2V617F allele burden was only possible in the same one patient $(0.95 \%$ of leukocytes, increase of $0.08 \%$ ). Hematological parameters remained remarkably stable with regard to initial values, and none of the patients had developed overt MPN according to the WHO criteria (Table 1). One patient (21484) had had a recurrent DVT during the interval.

\section{Discussion}

The JAK2V617F mutation was observed in 2.3\% (95\% CI 0.1-4.4) of patients with objectively diagnosed DVT of the leg and in one control without DVT $(0.5 \%$; $95 \%$ CI -0.5-1.5). The low prevalence of JAK2V617F among patients with venous thrombosis outside the splanchnic area in the absence of MPN has been reported before $(0.0-2.0 \%)$ [18-22], but not in a distinct cohort with DVT of the leg as in our study. Moreover, the presence of the JAK2V617F mutation increased the risk for DVT 4.5-fold (OR 4.5; 95\% CI 0.5-40.9), although not statistically significant. None of our JAK2V617F-positive patients developed overt MPN after a median follow-up of 68.5 months. Therefore, we conclude that in patients with venous thrombosis outside the splanchnic area, presence of the JAK2V617F mutation appears not to be clinically relevant.

To date, little is known about the clinical relevance of JAK2V617F presence in patients without overt MPN. It is 
Table 1 Characteristics and hematological parameters of JAK2V617F-positive patients at initial determination (1) and follow-up (2)

\begin{tabular}{|c|c|c|c|c|c|c|c|c|c|c|}
\hline & \multicolumn{10}{|c|}{ DNA sample ID } \\
\hline & \multicolumn{2}{|c|}{6578 (DVT) } & \multicolumn{2}{|c|}{21484 (DVT) } & \multicolumn{2}{|c|}{25287 (DVT) } & \multicolumn{2}{|c|}{33266 (DVT) } & \multicolumn{2}{|c|}{26660 (control) } \\
\hline & 1 & 2 & 1 & 2 & 1 & 2 & 1 & 2 & 1 & 2 \\
\hline Age (years) & 40 & 46 & 69 & 76 & 43 & 49 & 85 & 89 & 64 & 70 \\
\hline $\operatorname{Sex}(M / F)$ & $\mathrm{F}$ & & M & & M & & $\mathrm{F}$ & & $\mathrm{F}$ & \\
\hline $\mathrm{Hb}(\mathrm{g} / \mathrm{dL})$ & 14.2 & 14.3 & 12.7 & 13.9 & 16.3 & 16.1 & 15.0 & 13.7 & 14.8 & 14.7 \\
\hline $\mathrm{Ht}(\mathrm{L} / \mathrm{L})$ & NA & 0.42 & 0.39 & 0.41 & NA & 0.46 & 0.43 & 0.43 & 0.44 & 0.43 \\
\hline WBC (10E9/L) & 9.4 & 9.3 & 9.6 & 4.3 & 5.5 & 6.8 & 7.7 & 10.3 & 6.7 & 7.8 \\
\hline Plt (10E9/L) & 294 & 259 & 172 & 182 & 277 & 215 & 305 & 372 & 233 & 223 \\
\hline JAK2V617F (\%) & $+(\mathrm{NQ})$ & $+(\mathrm{NQ})$ & $+(\mathrm{NQ})$ & $+(\mathrm{NQ})$ & $+(\mathrm{NQ})$ & Neg & $+(0.87)$ & $+(0.95)$ & $+(\mathrm{NQ})$ & $\mathrm{Neg}$ \\
\hline Follow-up (months) & 69 & & 85 & & 67 & & 47 & & 69 & \\
\hline Status & Alive & & Alive & & Alive & & Alive & & Alive & \\
\hline
\end{tabular}

$N A$ not available, $N Q$ not quantifiable

not clear whether these patients are at imminent risk for transformation into MPN or other hematological disorders. Long-term follow-up in patients with SVT in the absence of MPN is limited, but suggests subsequent development of MPN in a considerable proportion of patients, although certainly not in all $[12,14-17,26]$. A small follow-up study in patients with other types of VTE could not observe MPN transformation after a median follow-up of 40 months [19], similar to our study.

However, it is not clear whether different therapeutic measures should be taken for JAK2V617F positive patients in the absence of MPN, with (or without) thrombosis. It also remains unclear why specifically SVT is more strongly associated with the JAK2V617F mutation in comparison with other locations. Finally, it is not known whether early diagnosis of MPN, based on JAK2V617F presence, benefits the prognosis or treatment of these disorders. Follow-up of larger JAK2V617F-positive non-MPN cohorts, with different locations of thromboses, is necessary to clarify these issues.

Follow-up on JAK2V617F allelic burdens has not often been reported in VTE patients in the absence of known MPN. A study in SVT-patients without MPN upon diagnosis showed stability of the mutated allele proportion in the majority of patients [14], also despite progression to MPN. We could not detect the JAK2V617F mutation upon reassessment after a median follow-up of 68.5 months in two of five initially positive patients. This could be explained by the insufficient presence of JAK2V617F in the proportion of examined cells, making the mutation undetectable at that instance, considering that the amount of mutated cells was already at the detection threshold of our sensitive assay initially. It could also be explained by disappearance of this small clone over time. However, the prognostic value of disappearing mutated clones is not yet known, nor in the context of treatment with hydroxyurea [6].

In general, prevalence assessment in all JAK2V617F studies is much dependent on PCR assay heterogeneity [25, $27]$ and the clinical diagnostic positivity cutoff value. Some studies commit to a clinical positivity cutoff of $1 \%$ of mutated cells for diagnostic MPN implications [27, 28]. The analytical threshold of our assay was $0.1 \%$, with DNA samples considered JAK2V617F positive upon duplicate positive PCR signals with $\mathrm{Ct}<43$, while an allelic burden of $1 \%$ results in a $\mathrm{Ct}$ value of 35 . Hence, samples can be considered JAK2V617F-positive without an available quantification of the mutated allele burden.

Highly sensitive assays like ours are necessary to determine minimal residual disease in patients following treatment for their MPN [6, 7]. However, it can also lead to positive results with low allele burdens when used for JAK2 detection in VTE patients or healthy subjects [9, 11, 27, 28]. These results should be interpreted with caution. Although some studies suggest that JAK2V617F in these patients may indicate an underlying MPN [9-11], the value of its presence in these patients, especially with low mutational levels, remains unclear. In our study, none of the JAK2V617F-positive patients developed a clinical diagnosis of MPN after a median follow-up of nearly 6 years. Therefore, we conclude that presence of the JAK2V617F mutation in non-MPN patients with venous thrombosis outside the splanchnic area appears not to be clinically relevant. 
Acknowledgments The authors would like to thank Joep Defesche, head of the DNA laboratory, Department of Vascular Medicine, Academic Medical Center, for providing the utilities to store DNA samples.

Disclosure There is no conflict of interest relevant to this manuscript for any of the authors.

Open Access This article is distributed under the terms of the Creative Commons Attribution Noncommercial License which permits any noncommercial use, distribution, and reproduction in any medium, provided the original author(s) and source are credited.

\section{Reference list}

1. Tefferi A, Elliott M (2007) Thrombosis in myeloproliferative disorders: prevalence, prognostic factors, and the role of leukocytes and JAK2V617F. Semin Thromb Hemost 33(4):313-320

2. Baxter EJ, Scott LM, Campbell PJ, East C, Fourouclas N, Swanton S et al (2005) Acquired mutation of the tyrosine kinase JAK2 in human myeloproliferative disorders. Lancet 365 (9464):1054-1061

3. James C, Ugo V, Le Couedic JP, Staerk J, Delhommeau F, Lacout $\mathrm{C}$ et al (2005) A unique clonal JAK2 mutation leading to constitutive signalling causes polycythaemia vera. Nature 434 (7037):1144-1148

4. Kralovics R, Passamonti F, Buser AS, Teo SS, Tiedt R, Passweg JR et al (2005) A gain-of-function mutation of JAK2 in myeloproliferative disorders. N Engl J Med 352(17):1779-1790

5. Tefferi A, Vardiman JW (2008) Classification and diagnosis of myeloproliferative neoplasms: The 2008 World Health Organization criteria and point-of-care diagnostic algorithms. Leukemia 22:14-22

6. Girodon F, Schaeffer C, Cleyrat C, Mounier M, Lafont I, Santos FD et al (2008) Frequent reduction or absence of detection of the JAK2-mutated clone in JAK2V617F-positive patients within the first years of hydroxyurea therapy. Haematologica 93(11):1723-1727

7. Kroger N, Badbaran A, Holler E, Hahn J, Kobbe G, Bornhauser M et al (2007) Monitoring of the JAK2-V617F mutation by highly sensitive quantitative real-time PCR after allogeneic stem cell transplantation in patients with myelofibrosis. Blood 109(3):1316-1321

8. Tefferi A, Gilliland DG (2005) JAK2 in myeloproliferative disorders is not just another kinase. Cell Cycle 4(8):1053-1056

9. Sidon P, El Housni H, Dessars B, Heimann P (2006) The JAK2V617F mutation is detectable at very low level in peripheral blood of healthy donors. Leukemia 20(9):1622

10. Nielsen C, Birgens HS, Nordestgaard BG, Kjaer L, Bojesen SE (2011) The JAK2 V617F somatic mutation, mortality and cancer risk in the general population. Haematologica 96(3):450-453

11. Xu X, Zhang Q, Luo J, Xing S, Li Q, Krantz SB et al (2007) JAK2(V617F): Prevalence in a large Chinese hospital population. Blood 109(1):339-342

12. Patel RK, Lea NC, Heneghan MA, Westwood NB, Milojkovic D, Thanigaikumar $\mathrm{M}$ et al (2006) Prevalence of the activating JAK2 tyrosine kinase mutation $\mathrm{V} 617 \mathrm{~F}$ in the Budd-Chiari syndrome. Gastroenterology 130(7):2031-2038

13. Smalberg JH, Murad SD, Braakman E, Valk PJ, Janssen HL, Leebeek FW (2006) Myeloproliferative disease in the pathogen- esis and survival of Budd-Chiari syndrome. Haematologica 91 (12):1712-1713

14. Kiladjian JJ, Cervantes F, Leebeek FW, Marzac C, Cassinat B, Chevret $S$ et al (2008) The impact of JAK2 and MPL mutations on diagnosis and prognosis of splanchnic vein thrombosis: a report on 241 cases. Blood 111(10):4922-4929

15. Colaizzo D, Amitrano L, Tiscia GL, Scenna G, Grandone E, Guardascione MA et al (2007) The JAK2 V617F mutation frequently occurs in patients with portal and mesenteric venous thrombosis. J Thromb Haemost 5(1):55-61

16. De Stefano V, Fiorini A, Rossi E, Za T, Farina G, Chiusolo P et al (2007) Incidence of the JAK2 V617F mutation among patients with splanchnic or cerebral venous thrombosis and without overt chronic myeloproliferative disorders. J Thromb Haemost 5 (4):708-714

17. Regina S, Herault O, D'Alteroche L, Binet C, Gruel Y (2007) JAK2 V617F is specifically associated with idiopathic splanchnic vein thrombosis. J Thromb Haemost 5(4):859-861

18. Pardanani A, Lasho TL, Schwager S, Finke C, Hussein K, Pruthi RK et al (2007) JAK2V617F prevalence and allele burden in nonsplanchnic venous thrombosis in the absence of overt myeloproliferative disorder. Leukemia 21(8):1828-1829

19. Pardanani A, Lasho TL, Hussein K, Schwager SM, Finke CM, Pruthi RK et al (2008) JAK2V617F mutation screening as part of the hypercoagulable work-up in the absence of splanchnic venous thrombosis or overt myeloproliferative neoplasm: assessment of value in a series of 664 consecutive patients. Mayo Clin Proc 83 (4):457-459

20. Remacha AF, Estivill C, Sarda MP, Mateo J, Souto JC, Canals C et al (2007) The V617F mutation of JAK2 is very uncommon in patients with thrombosis. Haematologica 92(2):285-286

21. Ugo V, Le GG, Lecucq L, Mottier D, Oger E (2008) Prevalence of the JAK2 V617F mutation is low among unselected patients with a first episode of unprovoked venous thromboembolism. J Thromb Haemost 6(1):203-205

22. Za T, Fiorini A, Rossi E, Ciminello A, Chiusolo P, Leone $\mathrm{G}$ et al (2009) Prevalence of the JAK2 V617F mutation in patients with unprovoked venous thromboembolism of common sites and without overt myeloproliferative neoplasms. Br J Haematol 144 (6):965-967

23. Wells PS, Anderson DR, Bormanis J, Guy F, Mitchell M, Gray L et al (1997) Value of assessment of pretest probability of deep-vein thrombosis in clinical management. Lancet 350 (9094):1795-1798

24. Quentmeier H, MacLeod RA, Zaborski M, Drexler HG (2006) JAK2 V617F tyrosine kinase mutation in cell lines derived from myeloproliferative disorders. Leukemia 20(3):471-476

25. Lippert E, Girodon F, Hammond E, Jelinek J, Reading NS, Fehse $B$ et al (2009) Concordance of assays designed for the quantification of JAK2V617F: a multicenter study. Haematologica 94(1):38-45

26. Dentali F, Squizzato A, Brivio L, Appio L, Campiotti L, Crowther $\mathrm{M}$ et al (2009) JAK2V617F mutation for the early diagnosis of $\mathrm{Ph}$ - myeloproliferative neoplasms in patients with venous thromboembolism: a meta-analysis. Blood 113(22):5617-5623

27. Rapado I, Albizua E, Ayala R, Hernandez JA, Garcia-Alonso L, Grande S et al (2008) Validity test study of JAK2 V617F and allele burden quantification in the diagnosis of myeloproliferative diseases. Ann Hematol 87(9):741-749

28. Martinaud C, Brisou P, Mozziconacci MJ (2010) Is the JAK2 (V617F) mutation detectable in healthy volunteers? Am J Hematol 85(4):287-288 